Louisiana State University

LSU Digital Commons

1970

\title{
Amplitude Effects for the Oscillating Disk in Liquid Helium-li.
}

Robert Patrick Roger

Louisiana State University and Agricultural \& Mechanical College

Follow this and additional works at: https://digitalcommons.Isu.edu/gradschool_disstheses

\section{Recommended Citation}

Roger, Robert Patrick, "Amplitude Effects for the Oscillating Disk in Liquid Helium-li." (1970). LSU

Historical Dissertations and Theses. 1747.

https://digitalcommons.Isu.edu/gradschool_disstheses/1747

This Dissertation is brought to you for free and open access by the Graduate School at LSU Digital Commons. It has been accepted for inclusion in LSU Historical Dissertations and Theses by an authorized administrator of LSU

Digital Commons. For more information, please contact gradetd@lsu.edu. 
$70-18,555$

ROGER, Robert Patrick, 1942-

AMPLITUDE EFFECTS FOR THE OSCILLATING DISK IN LIQUID HeII.

The Louisiana State University and Agricultural and Mechanical College, Ph.D., 1970 Physics, general

University Microfilms, Inc., Ann Árbor, Michigan 
AMPLITUDE EFFECTS FOR THE

OSCILLATING DISK IN LIQUID HEII

\author{
A Dissertation \\ Submitted to the Graduate Faculty of the \\ Louisiana State University and \\ Agricultural and Mechanical College \\ in partial fulfillment of the \\ requirements for the degree of \\ Doctor of Philosophy \\ in
}

The Department of Physics and Astronomy

by

Robert Patrick Roger

B.S., Loyol. University of the South, 1964

January 1970 


\section{ACKNOWLEDGEHENT}

The author wishes to express his gratitude to Dr. R. G. Hussey for suggesting the topic of investigation and for his assistance throughout the course of the research. The patience and encouragement displayed by my wife and family was a decisive influence and is gratefully appreciated.

The author acknowledges the skillful help of Leslie Edelen, Charles Burlo, and Heinz Eichenseer for their help with the equipment. The financial support of this research by the National Science Foundation under Contract No. GP-7109 is acknowledged and appreciated. The author also acknowledges the financial assistance received from the Dr. Charles E. Coates Memorial Fund of the L.S.U. Foundation donated by veorge H. Coates for the expenses of preparation of this dissertation. 


$\begin{array}{lc} & \text { Page } \\ \text { I. INTRODUCTION } & 1 \\ \text { II. EXPERIMENT } & 2 \\ \text { III. RESULTS } & 5 \\ \text { IV. DISCUSSION } & 10 \\ \text { V. CONCLUSIONS } & 17 \\ \text { REFERENCES } & 20 \\ \text { APPENDIX I } & \text { AI-1 } \\ \quad \text { CALCULATION OF LOW AMPLITUDE LOGARITHMIC } & \\ \text { DECREMENT } & \text { AII-1 } \\ \text { APPENDIX II } & \\ \quad \text { COMPUTER PROGRAM USED TO CALCULATE ANO PLOT } & 23 \\ \text { VITA }\end{array}$




\section{LIST OF TABLES}

Table

Page

1. Experimental Disks 3a

11. Parameters in the Calculation of Energy Dissipation Due to Mutual Friction $16 a$

111. Values of Normal Fluid Viscosity and Density At-2

IV. Calculated and Observed Volues of Logarithmic Decrement at Low Ampli tude

$A \mid-3$ 


\section{LIST OF FIGURES}

Figure

Page

1. Experimental Cryostet

2. Logarithmic Decrement vs. Amplitude for a Steep and Flat Curve

3. Flet Damping Curves for Different Temperatures

$7 a$

4. High Amplitude Correlation for Flat Curves

$7 b$

5. Steep Damping Curves for Different Temperatures

$7 c$

6. High Amplitude Correlation for Steep Curves

7. Critical Velocities for Flat Curves

$8 \mathbf{8}$

8. Critical Velocities for Steep Curves

$8 b$

9. Amplitude Dependence of the Relative Change In Ia for Both $S$ teep and Flat Curves for Two Different Temperatures for a $3.9 \mathrm{sec}$ Perlod

10. Amplitude Dependence of the Relative Change in Ia for Both Steep and Flat Curves at one Temperature for a 11.54 sec Perlod

11. Depth Dependence of Low Amplitude Demping

12. Comparison of Excess Damping with Zwanikken's Theory 


\section{ABSTRACT}

The amplitude variation of the logarithmic decrement 0 of a disk (radius $\approx 2.5 \mathrm{~cm}$ ) performing torsional oscillations in liquid He If has been found to depend on the history of the hellum bath. Two types of damping curves are observed: (1) The first (called "steep") occurs when the height, $h$, of the beth above the disk is greater than some critical height, $h^{\prime},\left(15.8 \leq h^{\prime} \leq 16.8 \mathrm{~cm}\right)$ and the time interval $\Delta \mathrm{t}$ between reaching the lambda temperature $T_{\lambda}$ and the initial deflection of the disk is short $(\Delta t<30 \mathrm{~min})$; (2) The second (called "flat") occurs when either $h<h$ ', or If $h>h^{\prime}$ then $\Delta t$ must be long $(\Delta t>60 \mathrm{~min})$. There is a critical velocity $v_{c}$ essociated with each type of curve. For the steep curves $v_{\mathrm{cl}}$ increases with temperature (from 1.33 to $2.15^{\circ} \mathrm{K}$ ) and varles with period os $T^{-\frac{1}{2}}$ for periods between $3.9 \sec$ and $19.6 \mathrm{sec}$. For the flet curves $v_{\mathrm{c} 2}$ is independent of temperature (from 1.4 to $2.156^{\circ} \mathrm{K}$ ), is larger than $v_{c l}$, and has a period dependence given by $v_{c 2} \tau^{-\frac{1}{2}}=1.4$ (cgs units) for periods between 3.9 sec and 45.0 sec. The difference between steep and flat curves is attributed to the presence of a long-lived motion of the superfluid (possibly an ordered erray of quentized vortex lines) In the subcritical flow for the steep case and the absence of this motion for the flat case. The low emplitude logar Ithmic decrement $D_{0}$ is found to be the same for the steep and flet curves. The ralative chenge in $D_{0}$ from the celculeted value $\left(\left(D_{0}-D_{\text {celc }}\right) / D_{0}\right)$ is found to increase as $h / \lambda$ decreases $(\lambda$ is the 
penotration depth). The excess demping slightly above $v_{c}$ cannot be explained by the effect of mutual friction. The relative change in moment of Inertia (1, $\left.1_{a 0}\right) / 1_{\text {ao }}$ at high amplitudes (up to $2.5 \mathrm{rad}$ ) indicates that for the steep curves about $30 \%$ of the superfluid is obteined for 4 sec period and $5 \%$ for $12 \mathrm{sec}$ period. No entraiment is indlcated for the flat curves. 


\section{INTRODUCTION.}

When a disk oscillates in liquid helium II at very low amplitudes, the logarithmic decrement $D_{n}$ is independent of amplitude and $c$ an be used to determine $\eta_{h} \rho_{n}$, the product of normal fluid viscosity and density. Hollis-Hallett was the first to report that above a certain critical amplitude, $\varphi_{c}$, the logarithmic decrement increases with amplitude; the critical velocity is then taken to be the velocity of the edge of the disk:

$$
v_{c}=2 \pi \varphi_{c} R / T
$$

where $R$ is the disk radius and $\tau$ is the period.

Experiments with oscillating disks, cylinders, and spheres have shown agreement on the following results: (1) the critical velocity varies $w i$ th temperature as $\rho_{s}^{-n}(0.25 \leq n \leq 0.5), 1,2,3,4$ where $\rho_{s}$ is the superfluid density; (2) roughness of the oscillating boundary surface lowers the value of $v_{c} i^{2,3}$ (3) the excess decrement $\left(0-D_{n}\right)$ above $\varphi_{c}$ varies approximately as $\rho_{s}{ }^{*}, 2$ A major point of disagreement is the period dependence of the critical velocity: some investigators have found $v_{c} \propto \tau^{-1 / 2}, 1,3$ and others have found $v_{c}$ to be independent of period., 4 other results of oscillating boundary experiments are the following: (1) $v_{c}$ depends on the size of the helium bath; ${ }^{4}$ (2) the effective density at high amplitudes (as revealed by an oscillating plle of. disks) exceeds $\rho_{n}$ and may approach the total density $\rho\left(-\rho_{s}+\rho_{n}\right)^{1}$; 
(3) the effective kinematic viscosity at high amplitude is less than $\eta_{n} / \rho_{n} \cdot 5$

In 1958 Donnelly and Hollis-Hallett ${ }^{6}$ reviewed experiments of this type and succeeded in associating a similarity parameter with $v_{c}$. Hall ${ }^{7}$ has connected this similarity parameter with superfluid turbulence by assuming that $v_{c}$ occurs when the average spacing between quantized vortex lines becomes of the order of the penetration depth $\lambda$ [defined as $\left.\left(\eta_{n} \tau / \pi \rho_{n}\right)^{\frac{1}{2}}\right]$. However, both of these results depend upon the critical velocity varying as $\tau^{-\frac{1}{2}}$. We have measured the amplitude dependence of the logarithmic decrement of an oscillating disk over a wider range of periods (3.9 to $45.0 \mathrm{sec)}$ than before. Our major results are: (1) both $v_{c}$ and the shape of the damping curves are strongly affected by the previous history of the helium bath; (2) when the helium bath is undisturbed for a sufficient time (about one hour) after lowering the temperature below the lambda point, $v_{c}$ is independent of temperature and varies as $\tau^{-\frac{1}{2}}$.

\section{I. EXPERIMENT}

The torsion pendulun cryostat used in this investigation is shown in Fig. 1. Both ends of the quartz torsion fiber A were glued into half inch pieces of No. 23 gauge needle with Aroldite epoxy. The needle on the upper end of the fiber was then cemented into a piece of No. 8 gauge needle which was fastened to the head piece $B$. The head, which was used to adjust the zero, was held vacuum tight by an o-ring connection to the vertically adjustable sleeve $C$, which 


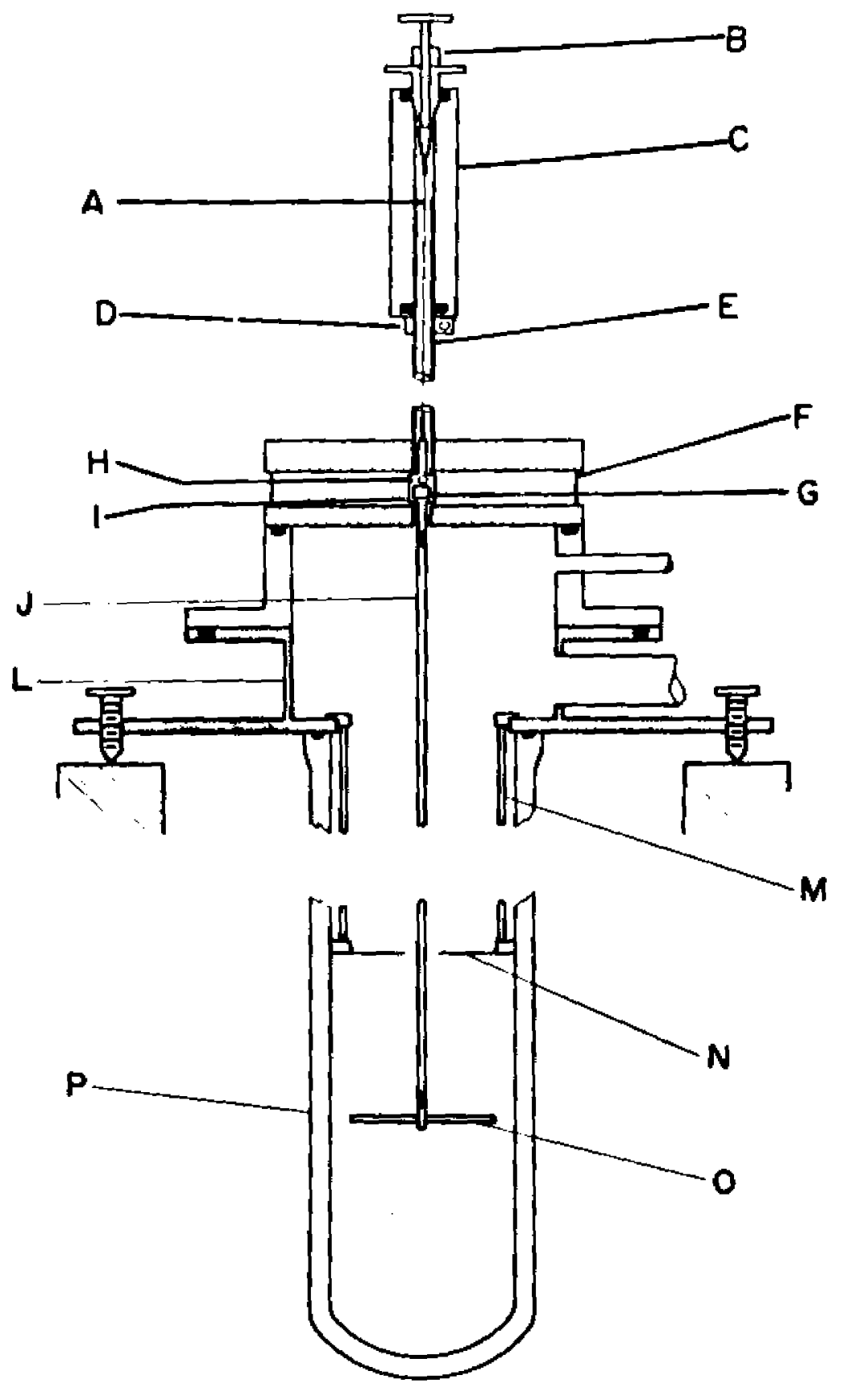

Fig. 1 
was fixed in a given position on the stainless steel tube $E$ by the clamp $D$. The lower end of the quartz fiber was similarly connected to the aluminum mirror mount 1 . The front surface mirror $G$ was placed so that its reflecting side lay on the axis of rotation. Just above the mirror, in the center of the mount, was placed a cylindrical piece of magnetized steel $\mathrm{H}$, which was used in connection with a set of external Helmholtz coils to deflect the system to a desired amplitude. After the initial deflection the Helmholtz coils were removed. To the bottom of the mirror mount was cemented a long glass tube $J$ of length $80 \mathrm{~cm}$ and outside diameter $0.31 \mathrm{~cm}$. The lower end of the tube was fastened to the disk 0 . Three highly polished disks were used (see Table 1). The disk could be pulled up to the shield $\mathrm{N}$ by means of the sleeve $\mathrm{C}$. This was done to prevent the surface of the disk from being contaminated with frost or dust particles during the precooling and transferring processes. The shield is a piece of aluminum foil which extends to the walls of the glass dewar $P$ and has a $13 \mathrm{~mm}$ diameter hole in its center. At its outer edge, the shield is attached to a brass $r i n g$ which is hung by three thin-walled stainless 5 teel tubes $M$ (o.d. $1.5 \mathrm{~mm}$ ) from the pumping collar $L$. When the disk was lowered, it was $4.6 \mathrm{~cm}$ from the shield and $10.5 \mathrm{~cm}$ from the bottom of the helium flask. The inside diameter of the helium flask was $7.0 \mathrm{~cm}$.

The amplitude of oscillation was measured by reading the leading edge of the focused image of a straight-filament light source on a circular scale of radius $1 / 3 \mathrm{~m}$. The viewing chamber $F$ 
Table I, Experimental Disks

$\begin{array}{ccccc}\text { Disk } & \begin{array}{c}\text { Radius } \\ (\mathrm{cm})\end{array} & \begin{array}{c}\text { Thickness } \\ (\mathrm{cm})\end{array} & \begin{array}{c}\text { Moment of Inertia } \\ \left(\mathrm{g} \mathrm{cm}^{2}\right)\end{array} & \text { Material } \\ \text { A } & 2.5103 & 0.1566 & 82.576 & \text { Brass } \\ \text { B } & 2.5318 & 0.2976 & 161.164 & \text { Brass } \\ \text { C } & 2.5307 & 0.1584 & 28.428 & \text { Aluminum }\end{array}$

These are thermally contracted dimensions. 
was turned down from a Plexiglas tube to a thickness of $0.0794 \mathrm{~cm}$ and radius of $4.85 \mathrm{~cm}$. The width of the light image on the scale was $0.75 \mathrm{~mm}$. A similar light, focused onto a slit placed in front of a photomultiplier tube, was used to measure the period of oscillation. The width of the light at this slit was $1.5 \mathrm{~mm}$ and the slit was $0.5 \mathrm{~mm}$ wide. The distance from the mirror to the photomultiplier was $1.5 \mathrm{~m}$. The pulse from the photomultiplier was fed into an amplifjer-pulse shaper and a scale-of-two circuit (to eliminate the mid-period pulse). The resulting square wave pulses were differentiated and used to activate a l MHz time base counter. Period accuracy at low amplitudes varied from $0.1 \mathrm{msec}$ at the short periods to $1 \mathrm{msec}$ at the longer periods.

The temperature was regulated with an elastic membrane regulator of the Walker type, ${ }^{8}$ and was measured with an oil monometer. The oil used had a density of $0.981 \mathrm{~g} / \mathrm{cm}^{3}$.

Measurements were made of the amplitude dependence of the logarithmic decrement and perlod of each torsion fiber in vacuum $\left(\sim 2 \times 10^{-6} \mathrm{~mm} \mathrm{Hg}\right)$. The vacuum decrement for all fibers used was essentially independent of amplitude and ranged from 0.00096 for the thin fibers to 0.00007 for the thicker ones. The vacuum period varied with amplitude (at 1.5 rad the change was $100 \mathrm{msec}$ for a 12 sec period and less than $1 \mathrm{msec}$ for a $4 \mathrm{sec}$ period), but the change was reproduceable, so calibration curves were taken before and after each run and the liquid heli um data were corrected for the vacuum variation. Measurements of the period in the liquid were made by measuring single periods, and at the low amplitudes several periods 
were averaged and a smooth curve drawn through these points was used in analyzing the results. The damping was small enough so that the logarithmic decrement could be determined from the expression

$$
D=\left[\ln \left(\varphi_{i} / \varphi_{j}\right)\right] /(j-i)
$$

where $\varphi_{i}$ is the $i$ th amplitude. This expression is a factor $2 \pi$ larger than the one used by Hollis Hallett. ' For runs with the smallest damping, the number of amplitudes $(j-i)$ used in Eq. (2) ranged typically from 7 at the high amplitudes to 30 at the low amplitudes; for runs with larger damping, the range was typically 4 to 15. Values of the critical velocity determined from semi-log plots of $\varphi_{i}$ vs. $i$ were in very good agreement with those obtained from curves of D vs. $\varphi$. The logarithmic decrements measured in Hell ranged from 0.0022 for disk B to 0.045 for disk C.

\section{RESULTS}

\section{A. Damping Curves}

Figure 2 shows the amplitude dependence of the logarithmic decrement $D$ of disk $C$ taken on two separate days at approximately the same temperature. For convenience, we describe the upper curve as "steep" and the lower curve as "flat". As long as the temperature remained below the lambda temperature $T_{\lambda}$, the damping curves were all of the same type, i.e. ef ther all steep or all flat. We have found that the difference between steep and flat curves is not due to (1) roughness of the disk surface, (2) the manner of deflecting 
יָ

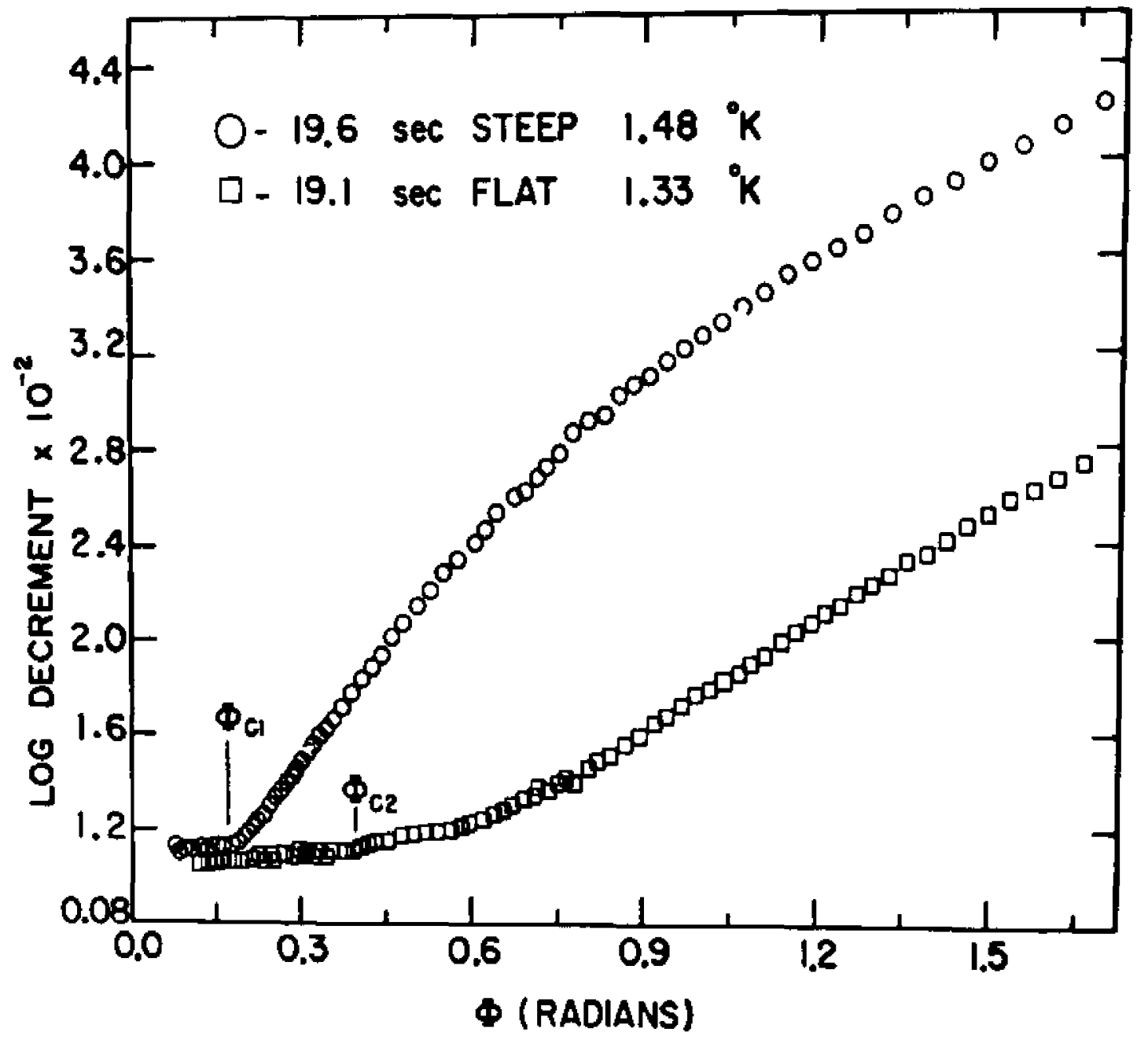


the disk (whether by many small impulses or by a few large impulses), or (3) the position of the disk as the temperature passed through $T_{\lambda}$ (whether lowered or held against the shield $N$ ). The difference is due to (1) the height of the bath at the time of the initial disk deflection, (2) the time interval $\Delta t$ between reaching $T_{\lambda}$ and the initial disk deflection, and possibly (3) the presence of the shield $N$. Using the cryostat in Fig. I (including shield N) we have found that in order to obtain a steep curve it is necessary that the bath height $h$ above the disk at the initial disk deflection be greater than some critical value $h^{\prime}$ and that $\Delta t$ be sufficiently short (probably less than $30 \mathrm{~min}$ ). To obtain a flat curve it is necessary to have $h<h^{\prime}$, or if $h>h^{\prime}$ then $\Delta t$ must be sufficiently long (about $l$ hour). The limits on $h$ ' have been found to be $15.8 \leq \mathrm{h}^{\prime} \leq 16.8 \mathrm{~cm}$. A few runs taken wi thout the shield indicate either that $h^{\prime}$ is lower in the absence of the shield (less than $10 \mathrm{~cm}$ ) or that perhaps the presence of the shield is necessary to get a flat curve. It should be pointed out, however, that once a steep or flat curve was established the lowering of the liquid level below the shield did not change the type of curve.

Occasionally, curves that were neither steep nor flat were observed. These curves were either in between the steep and flat curves ( 8 out of 125 curves), exhibiting a critical velocity in between those for steep and flat, or lower than the flat curves ("ultra-flat", 7 out of 125), four of which exhibited critical velocities in agreement with the flat curves and three which had 
critical velocities higher than the flat ones. However, there was no correlation between the occurrence of these odd curves and the time interval $\Delta t$; in particular, one ultra-flat curve was obtained with a $\Delta \mathrm{t}$ of only $10 \mathrm{~min}$. Of the 125 curves, $88 \%$ were either steep or flat. On three separate occasions, both steep and flat curves were obtained on the same day by warming above $T_{\lambda^{*}}$ it should be emphasized that the low amplitude damping was the same for both steep and flat curves.

Flat curves at different temperatures for an $11.54 \mathrm{sec}$ period are shown in Fig. 3. Above the critical amplitude the logarithmic decrement increases and eventually becomes linear in the amplitude up to the highest amplitudes measured $(2.5 \mathrm{rad})$. The slope ( $\equiv d D / d \varphi$ ) at the high amplitudes was found to vary as $\tau^{\frac{1}{2}} / I$ as shown in Fig. 4, where $I$ is the moment of inertia of the disk and $\tau$ is the period.

Steep curves at different temperatures for an $11.54 \mathrm{sec}$ period are shown in Fig. 5. At the high amplitudes these curves also are linear in the amplitude; the slope varies as $\tau / I^{\frac{1}{2}} d$ as shown in Fig. 6, where $d$ is the thickness of the disk. Rosenblat ${ }^{9}$ has shown that the centrifugal forces on fluid near an oscillating disk cause a radial-axial secondary flow which makes the damping of the disk change with amplitude; such changes have recently been observed in classical fluids. ${ }^{10}$ The dashed curves in Fig. 3 and Fig. 5 indicate the changes expected for secondary flow of the normal fluid only, as given by the empirical results of Folse. 10 


$$
\varepsilon \cdot 6 !
$$

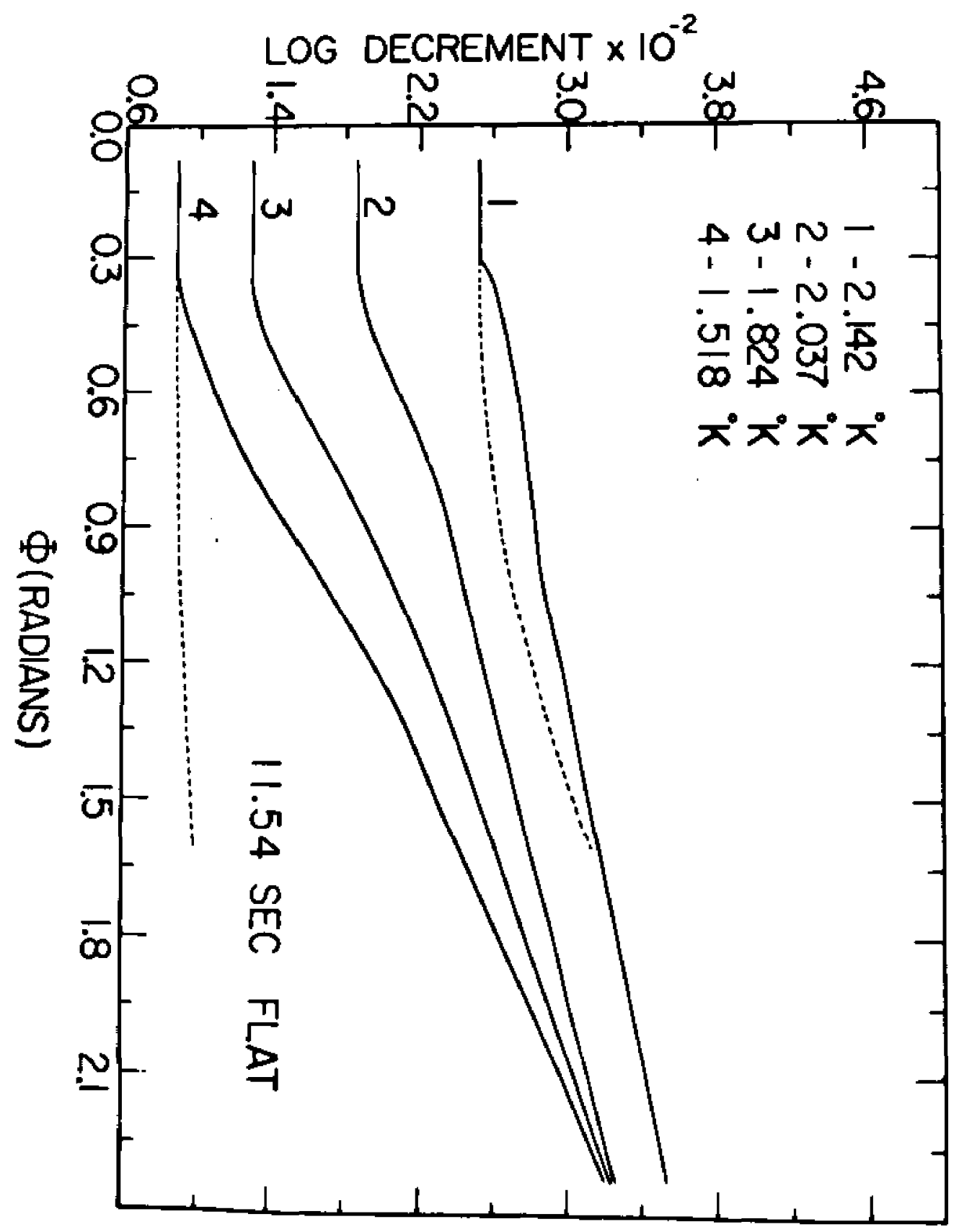

e $L$ 


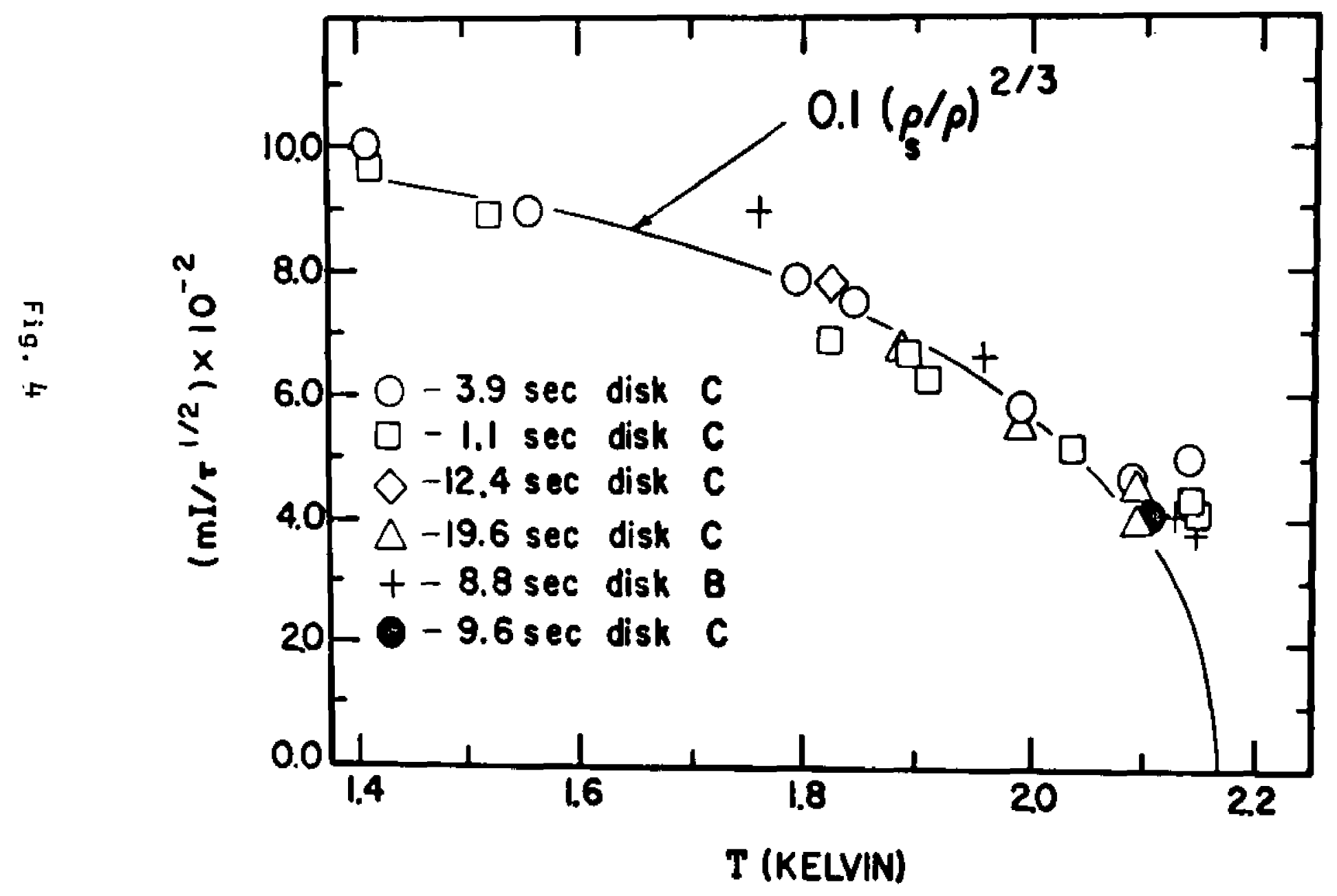




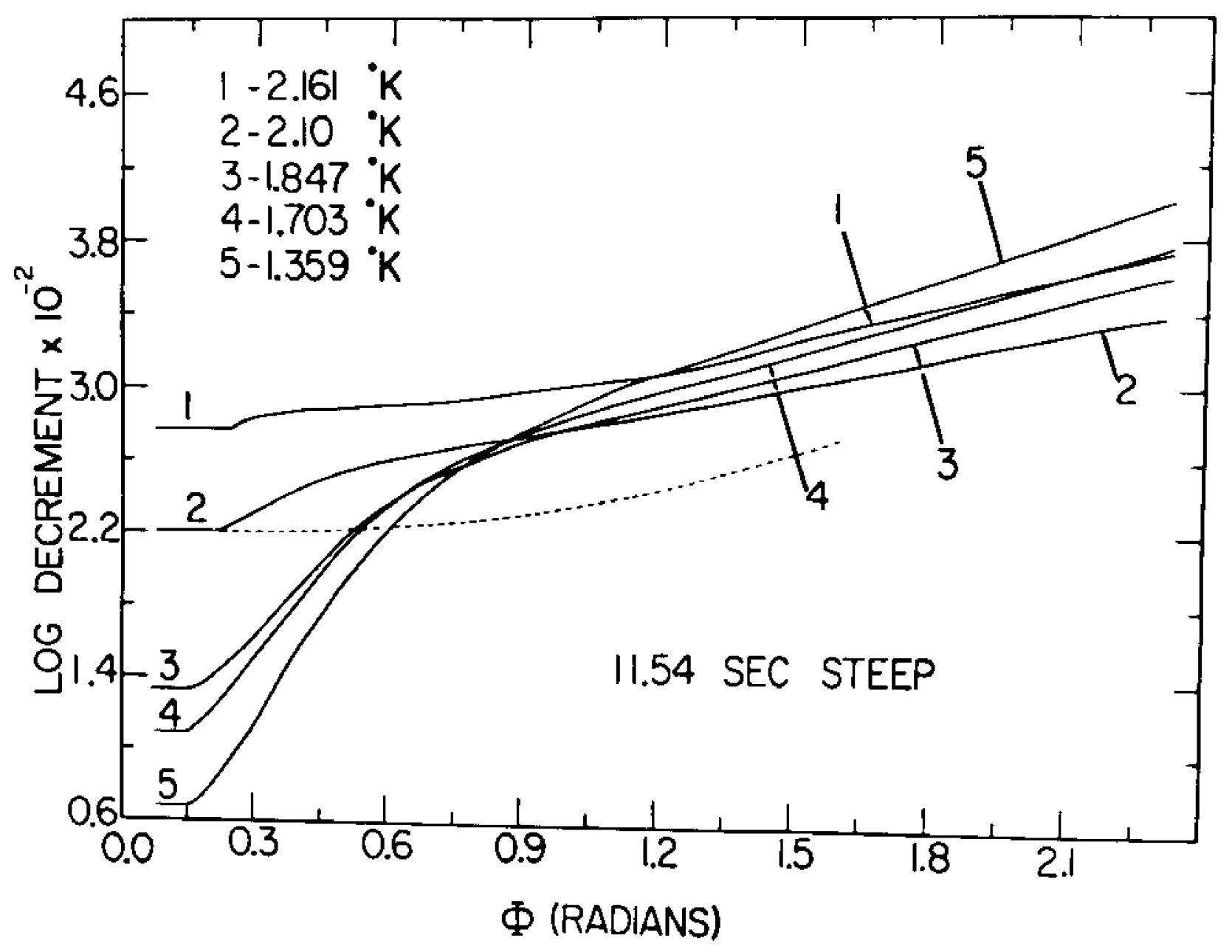




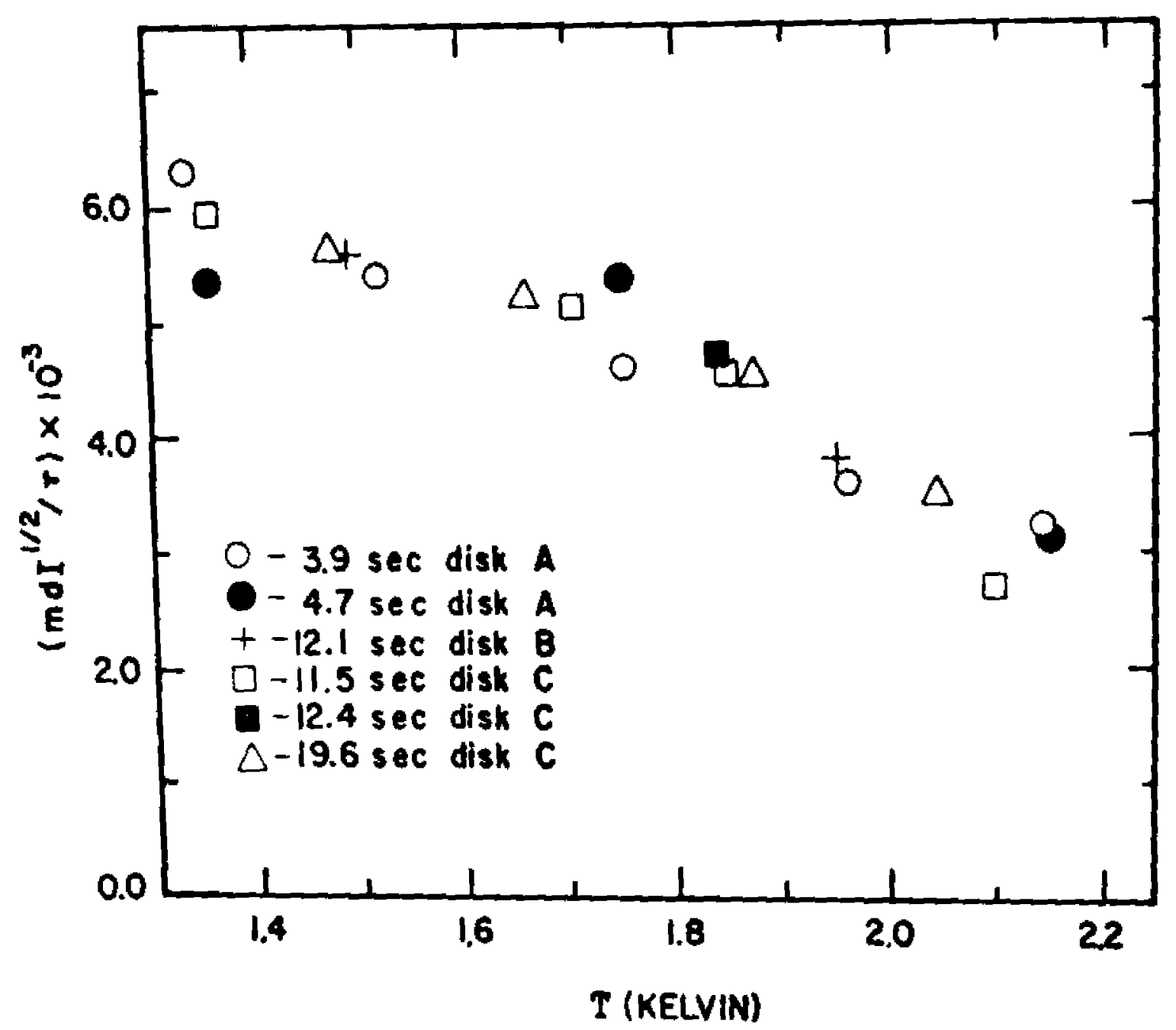


On several occasions we studied the effect of surface roughness by placing iron filings on the disk. For the steep curves, we found that surface roughness lowers the critical velocity and makes the high amplitude part of the damping curve steeper. There was some indication that surface roughness had no effect on the flat curves, but this result is, as yet, inconclusive.

B. Critical Velocities

The critical velocities $v_{c 2}$ associated with the flat curves are shown in Fig. 7. For periods between $3.9 \mathrm{sec}$ and $45.0 \mathrm{sec}$, $v_{c 2}$ is independent of temperature. The period dependence is given by $v_{\mathrm{c} 2}-1.4 \tau^{-\frac{1}{2}} \mathrm{~cm} / \mathrm{sec}$, which is represented by the solid lines in Fig. 7. The critical velocities $v_{c l}$ for the steep curves are shown in fig. 8. For periods between $3.9 \mathrm{sec}$ and $19.6 \mathrm{sec}$, we find that the period dependence at the lower temperatures is given by $v_{c l}=0.7 \tau^{-\frac{1}{2}} \mathrm{~cm} / \mathrm{sec}$. The results of Gamtsemlidze ${ }^{2}(\tau=8.95 \mathrm{sec})$ and Hollis Hallett ${ }^{1}(\tau=11.0 \mathrm{sec})$ are indicated by the broken line and the crosses respectively.

C. Period Measurements

The period of oscillation provides a measure of the added moment of inertia $I_{a}=\alpha\left(\tau^{2}-\tau_{v}{ }^{2}\right)$, where $\tau$ is the period of the disk ascillating in the liquid, $\tau_{v}$ is the period in vacuum, and $\alpha$ is the torsion constant. The relative change in $I_{a}$ with amplitude is then given by

$$
\left(I_{a}-I_{a o}\right) / I_{a o}=\left(\tau^{2}-\tau_{0}^{2}\right) /\left(\tau_{0}^{2}-\tau_{v}^{2}\right)
$$

where $I_{20}$ and $\tau_{0}$ are low amplitude values. Our results for a 3.9 sec 


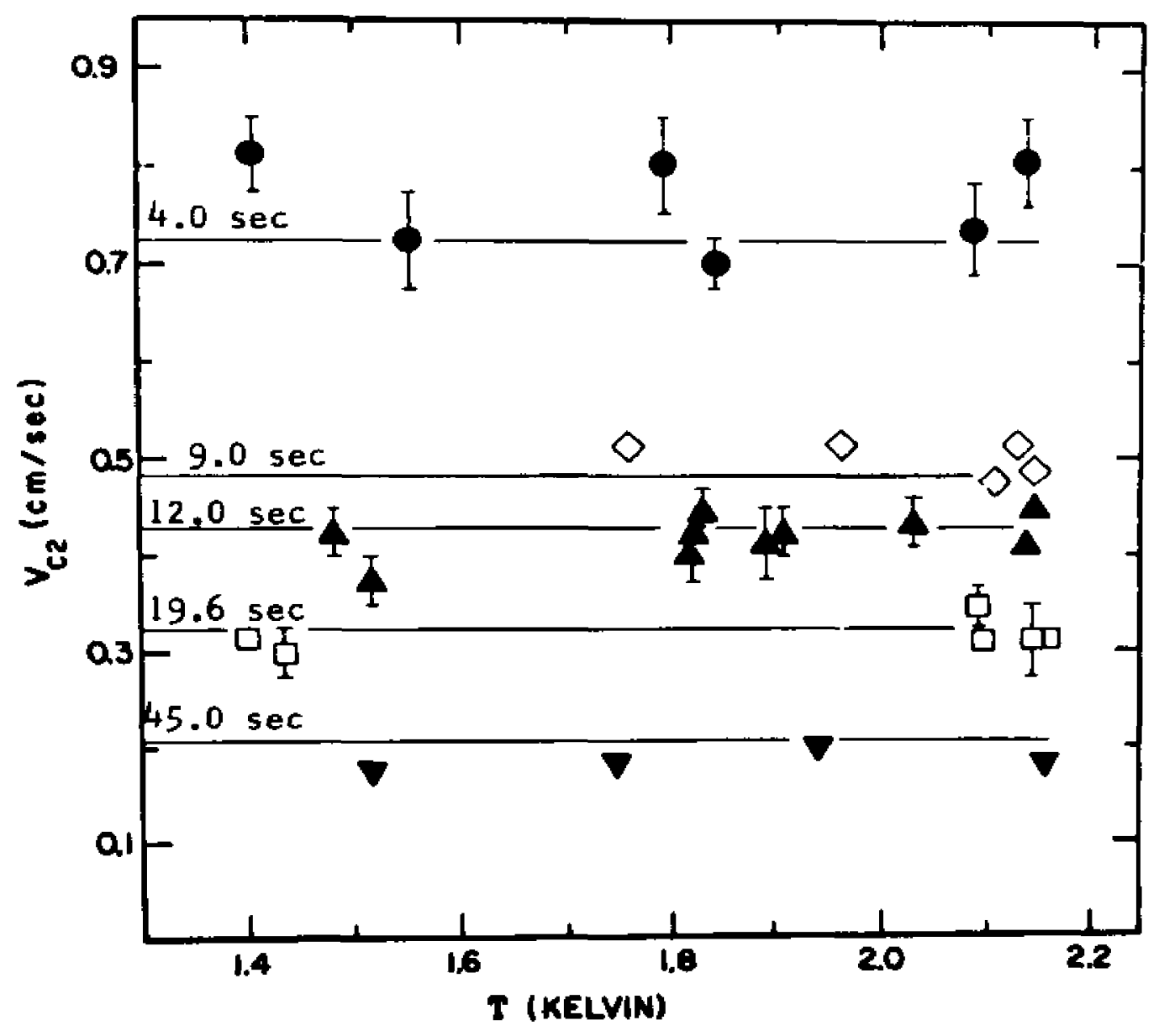

Fig. 7 
$\stackrel{7}{0}$

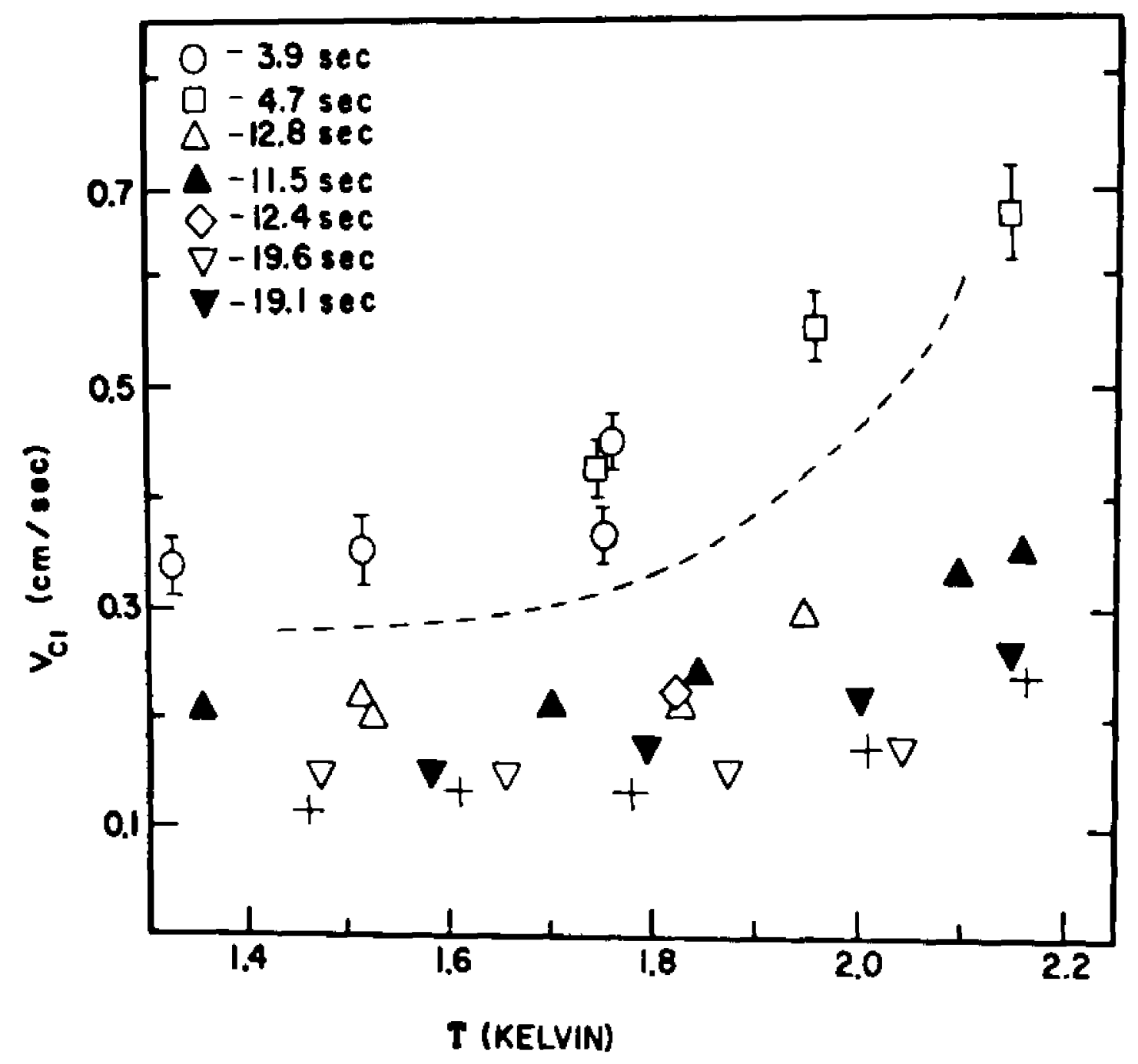


period at a high and low temperature for both steep and flat curves are shown in Fig. 9. The low temperature results can be compared with those in Fig. 10 for an $11.54 \mathrm{sec}$ period. In both figures the dashed curves represent what is expected from secondary flow of the normal fluid only. 10

D. Low Amplitude Damping

We have compared our observed logarithmic decrement $D_{0}$ at low amplitude with a calculated value $D_{\text {calc }}$ based upon $\eta_{n}$ from the rotating cylinder viscometer ${ }^{11}$ and $\rho_{n}$ from the second sound velocity. 12 The expression for $D_{\text {calc }}$ is the same as that used by Dash and Taylor, ${ }^{16}$ but with a corner parameter of $0.30 .^{13} \mathrm{in}$ general, $D_{c a l c}$ and $D_{0}$ agree within experimental error, but at low fluid depth and large penetration depth, $D_{0}$ is significantly higher. On several occasions with the helium at constant temperature, $D_{0}$ was observed to increase as the fluid level fell to within 100 to 200 penetration depths of the disk surface. Fig. 11 shows that $\left(D_{0}-D_{\text {calc }}\right) / D_{c a l c}$ depends only on the ratio $h / \lambda$, where $h$ is the fluid level above the disk and $\lambda$ is the penetration depth. The results in Fig. Il are independent of whether the damping curve is s teep or flat, or whether the shield was present, or which disk was used.

It appears that in superfluid helium the free surface can becone a serious source of energy loss in oscillating disk experiments when the fluid level is within a few hundred penetration depths. This result may explain the high values of $\eta_{n}$ obtained with a single disk ${ }^{16}$ (at temperatures below $1.5^{\circ} \mathrm{K}$ ) as compared with other 


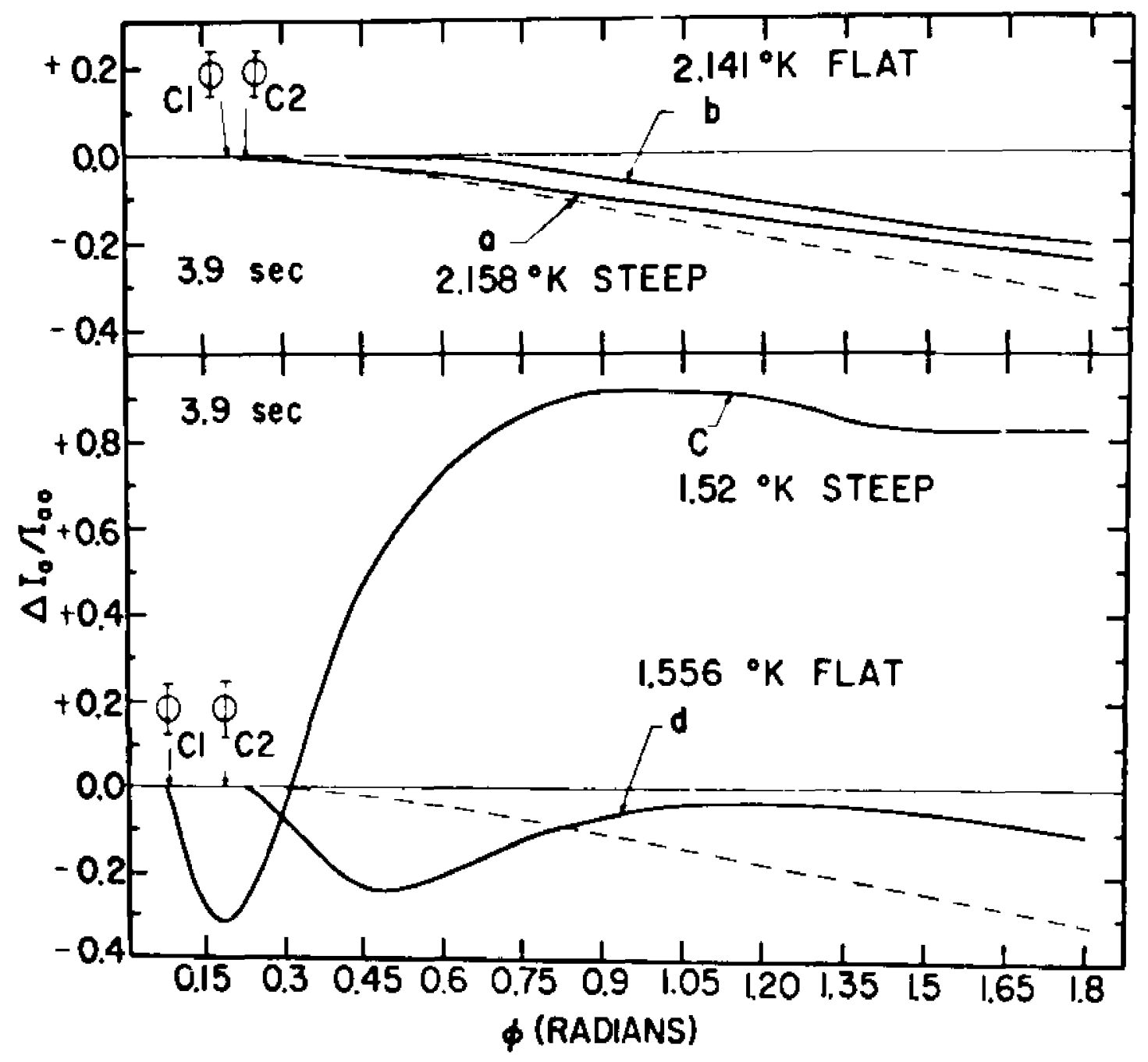




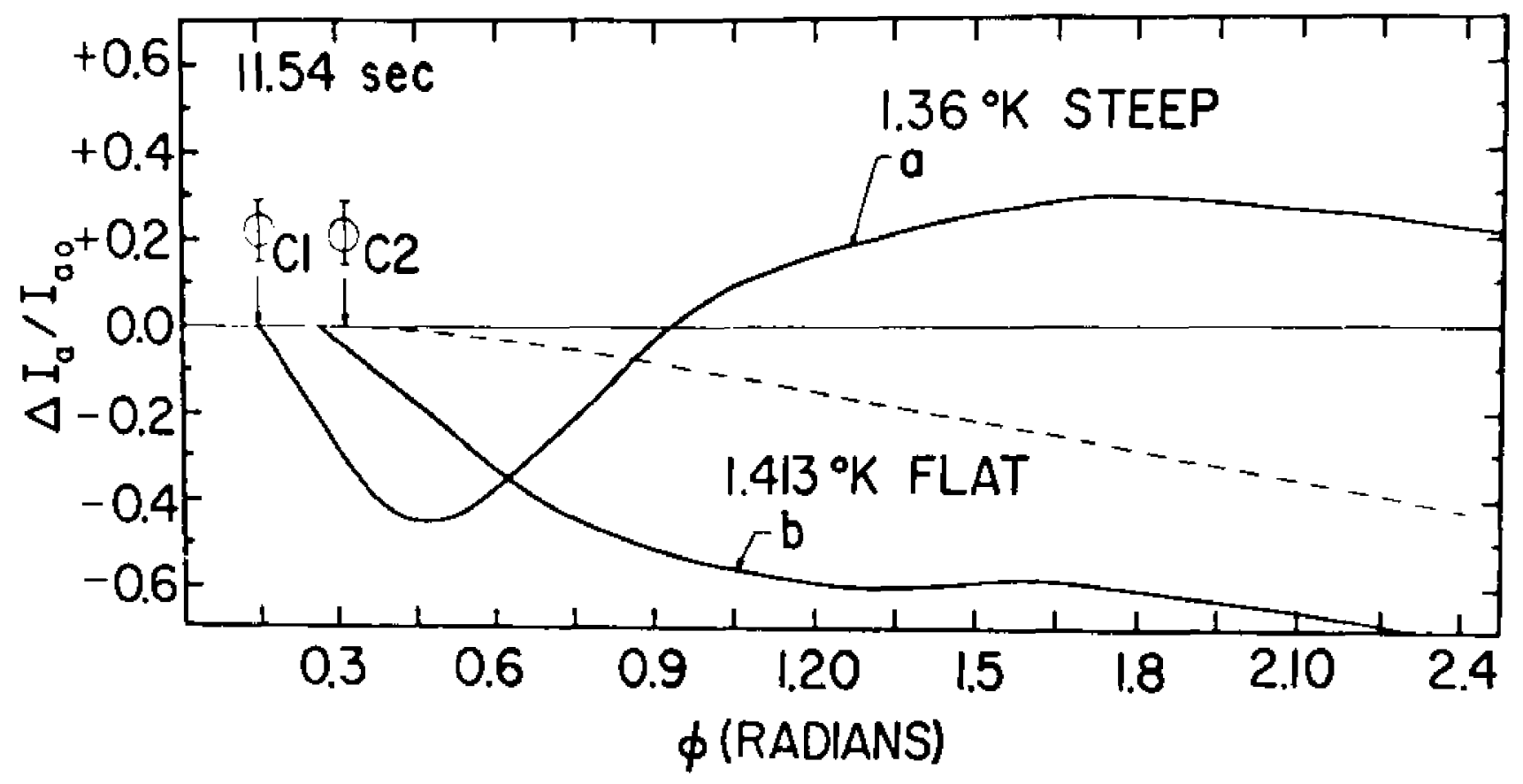


$n$
$\square$
$\square$

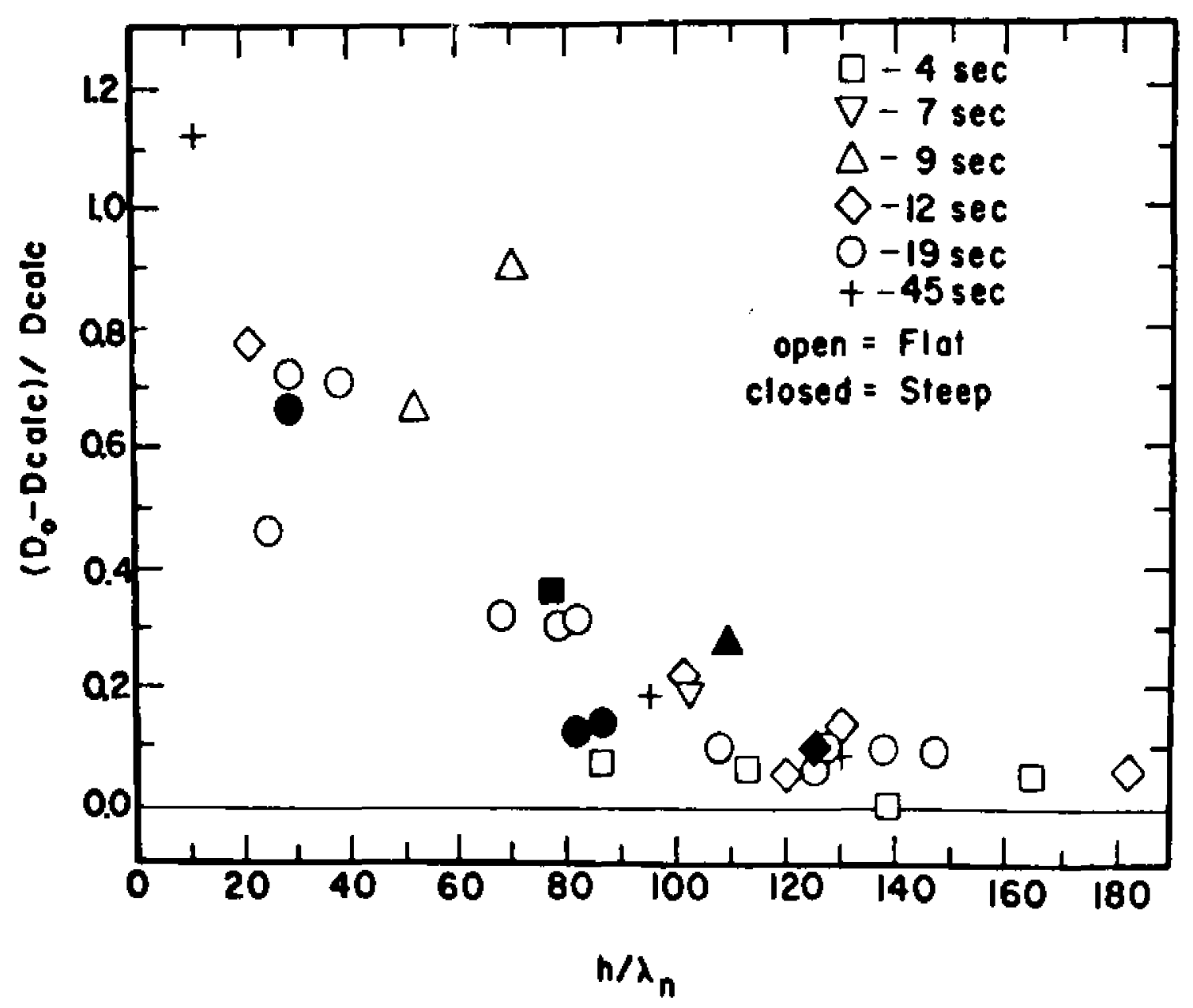

$\check{\circ}$ 
me thods. 14,11 Comparison with the results of Folse ${ }^{15}$ shows that this depth effect does not occur in classical liquids.

\section{DISCUSSION}

\section{A. Types of Damping Curves}

We will assume that the excess energy dissipation at high amplitudes originates from turbulence in the superfluid. Hall 7 and Vinen ${ }^{17-}$ have shown how the concept of superfluid turbulence can be introduced in the framework of a quantized vortex line mode $1.18,19$

In the present investigation we observe that the type of damping curve depends on the time interval $\Delta t$ from reaching the lambda point to the initial deflection of the disk. One explanation of this behavior is that the superfluid is created originally in a state of disorganized motion and if the system is undisturbed (the disk is not deflected) this random primordial flow eventually decays, leaving the superfluid relatively motionless, so that subsequent measurements produce flat curves; but if soon after reaching $T_{\lambda}$ the disk is deflected to high amplitudes, then the initial random motion of the superfluid is organized into a longlived state, and a steep curve results. However, since the low amplitude damping is the same for both steep and flat curves, this long-lived motion of the superfluid must not interact with the disk at low amplitude. In the framework of the vortex line picture, we could interpret the long-lived superfluid motion in the steep case as some sort of organized array of quantized vortex lines. It should be noted however, that whatever the subcritical vortex 
configuration is for the steep curves, it remains present for long periods of time (at least 8 hours for a series of curves and 70 min in between curves) and may persist indefinitely. We never observed a transition from one type of curve to another as long as the temperature remained below $T_{\lambda}$.

Results which depend on the previous history of the bath have been observed in a number of other liquid helium experiments, especially in those dealing with thermal counterflow in wide channels ${ }^{20-23}$ (diam $>10^{-3} \mathrm{~cm}$ ). All of the history effects in the thermal counterflow experiments were attributed to the presence of lingering vorticity in the superfluid. In the rotating beaker experiment of Reppy and Lane, ${ }^{24}$ two different results were obtained: usually the superfluid would absorb angular momentum from the coasting beaker, occasionally it would not; these results also were attributed to the presence or absence of primordial vortex lines.

Judging from the magnitude and temperature dependence of the critical velocities and the qualitative shape of the logarithmic decrement curves for a single disk observed by both Hollis-Hallett and Gamtsemlidze, ${ }^{2}$ we can classify their data in the steep category. However, some of the damping curves shown for a pile of disks in the pioneering work of Hollis-Hallett are qualitatively different, and may be of the flat type.

\section{B. Critical Velocities}

The temperature independence of $v_{c 2}$ (between 1.4 and $2.15^{\circ} \mathrm{K}$ ) reported here is in sharp disagreement with the $\rho_{s}^{-\frac{1}{2}}$ dependence found 
in most previous experiments of this type. However, there have been many other types of experiment which have yielded temperature independent critical velocities. For example, Ricci and Vicentini-Missoni ${ }^{25}$ have used a beam of ions to study heat flow in wide channels $(0.8 \mathrm{~cm})$ and have found critical velocities which were constant from 0.89 to $1.92^{\circ} \mathrm{K}$; Vermeer, Van Alphen, et. al. 26 have performed measurements of gravitational superfluid flow in wide channels with the normal fluid held motionless by means of superleaks and have found $v_{c}$ to be independent of temperature for a wide range of channel size (from 0.44 to $0.0015 \mathrm{~cm}$ ); Keller and Hamme $1^{27}$ have studied isothermal gravitational flow through two narrow slits $\left(2.29 \times 10^{-4} \mathrm{~cm}\right.$ and $\left.3.1 \times 10^{-5} \mathrm{~cm}\right)$ and have found $v_{c}$ to be independent of temperature except for a small region in the vicinity of $T_{\lambda}$ where $v_{c}$ falls rapidly to zero. Similar behavior is also found in film flow of HeII, 28,29 al though the results are complicated by uncertainties in the film thickness and the appropriate value of $\rho_{\mathbf{s}}$.

Keller and Hammel have observed that the narrow region $T_{\lambda}-T$, in which $v_{c}$ drops from its constant low-temperature value to zero at $T_{\lambda}$ ' decreases with increasing channel width; $c$ low and Reppy 30 have seen the same result with a superfluid gyroscope. In view of this it is understandable that we observe no decrease in $v_{c 2}$ up to the highest temperature $\left(2.156^{\circ} \mathrm{K}\right)$ since our smallest characteristic dimension was $\lambda \approx 0.014 \mathrm{~cm}$. Langer and $F$ isher 31 have developed a theory to explain this decrease of $v_{c}$ near $T_{\lambda}$ based on the idea that near $T_{\lambda}$ there should be an intrinsic critical velocity 
for the superfluid proportional to the superfluid density $\rho_{s}$. Vinen ${ }^{32}$ distinguishes between two types of critical velocity, an "ideal" type in which the subcritical superfluid flow is frictionless and free of vortex lines, and a "non-ideal" type in which vortex lines are present in the subcritical flow. One would expect that the non-ideal critical velocity would ordinarily be lower than the ideal type because the vortex lines in the subcritical region would act as nuclei for the growth of more line. However, it is difficult to determine what types of experiment give ideal critical velocities; persistent current and film flow experiments are suggested by Vinen. Since these experiments lead to critical velocities which are probably temperature independent (except near $T_{\lambda}$ ), it is possible that temperature independence may be an identifying feature of ideal critical velocities. If these ideas are correct, it is reasonable to identify the critical velocities obtained from our flat damping curves as ideal and those obtained from the steep damping curves as non-ideal.

Wilks has correlated critical velocities in wide channels $\left(>10^{-3} \mathrm{~cm}\right)$ by means of the expression

$$
v_{c}=(4 h / M d)\left[\ln \left(d / 3 a_{0}\right)\right]
$$

where $d$ is a characteristic length, $a_{0}=1.2 \times 10^{-8} \mathrm{~cm}$, and $M$ is the mass of a helium atom. Since $a_{0}$ is very small, the logarithmic factor is relatively insensitive to the value of $d$, so eq. (4) implies $v_{c} d$ const. Therefore, our result 
$v_{\mathrm{c} 2} \tau^{\frac{1}{2}}=1.4$ suggests that $d$ is a penetration depth based on an effective kinematic viscosity $\nu$ which is independent of temperature. The value of $\nu$ obtained in this manner is $\nu=1.2 \times 10^{-4} \mathrm{~cm}^{2} / \mathrm{sec}$, which is smaller than the normal fluid kinematic viscosity $\nu_{n}$. We associate with $v_{\mathrm{C} 2}$ a critical value of the Reynolds number Re. Re is defined by

$$
\operatorname{Re}=v \lambda / \nu=v(\tau / \pi \nu)^{\frac{1}{2}}
$$

and has a critical vlue of 72 .

Van Alphen et al. ${ }^{33}$ have proposed that in experiments where the normal fluid velocity $v_{n}$ is not negligible the critical velocities are related to classical turbulence in the normal fluid. They propose a Reynolds number Re $v_{n}-\frac{d \rho v_{n}}{\eta_{n}}$ which is based on the normal fluid viscosity $\eta_{n}$ and the total density $\rho$. Using $d=\left(\eta_{n} T / \pi \rho\right)^{\frac{1}{2}}$ and $v_{n}=v_{c 2}$, we find that the critical value of their Reynolds number ranges from 64 to 84 between $2.15^{\circ} \mathrm{K}$ and $1.3^{\circ} \mathrm{K}$. Our value of 72 is in good agreement, as would be expected since our effective $\nu$ is approximately equal to $\eta_{n} / \rho$. In spite of this agreement, we do not believe that a transition to classical turbulence takes place at this value of Re. We have made measurements in helium gas at $4.2^{\circ} \mathrm{K}$ and in liquid helium I at $2.4^{\circ} \mathrm{K}$ up to $\mathrm{Re}=430$ and have observed only gradual increases in damping consistent with the small increases expected from secondary flow. 10 The measurements of folse extended up to Re $=210$ in water; no sharp changes in damping were seen. A transition to turbulence would presumably be accompanied by sharp 
increases in damping above the critical amplitude such as those observed by Benson and Hollis Hallett ${ }^{3}$ for an oscillating sphere in helium gas $(\operatorname{Re}-230)$ and in helium $I(R e=210)$. Therefore, we conclude that although $R_{v_{n}}$ may be an important similarity parameter for liquid helium critical velocities, the critical value 72 does not correspond to classical turbulence in the normal fluid.

C. Other Results

(1) Effect of Mutual Friction

In 1950 Zwanikken ${ }^{33}$ derived an expression for the excess energy dissipation due to the mutual friction ${ }^{34}$ between the normal and superfluids in the case of the oscillating disk. Hollis-Hallett applied Zwanikken's analysis to his results and found the predicted energy dissipation to be smaller than observed and to have a different temperature variation. However, there appears to be a sign error in Hollis-Haliett's expression. Fur thermore, to apply Zwanikken's result to our disk requires more terms than orginally included because of the large radius of our disk. Therefore, we have extended Zwanikken's analysis and have found that the logarithmic decrement due to mutual friction can be written as

$$
D=A \rho_{s} \rho_{n} \lambda\left(\pi^{2} R^{4} / 8 I\right) \epsilon\left[1+\Phi_{1} \epsilon+\Phi_{2} \epsilon^{2}+\Phi_{3} \epsilon^{3}+\Phi_{4} \epsilon^{4}\right]
$$

where $A$ is the Gorter-Mellink mutual friction paraneter, $\lambda$ is the normal fluld penetration depth, $\epsilon$ is defined to be $\omega R^{2} \varphi^{2}$ ( $\varphi$ is 
the ampli tude), and the $\Phi_{i}$ terms depend only on temperature. Tabulated values of the major teriperature dependent terms are given in Table 11 . The values of $A$ were read from a drawing in a paper by Vinen. 23 Using the table and eq. 6 to calculate the damping at an amplitude just above the critical amplitude and comparing the result with our data we find that the calculated value is several orders of magnitude too large and highly temperature dependent. For example, at $\varphi=0.6 \mathrm{rad}$, $\tau=12 \mathrm{sec}$ and $T=1.9^{\circ} \mathrm{K}$, the calculated excess decrement is 1.76 and the observed value is 0.002 . However, if we try to obtain better agreement with the flat curve results by assuming that instead of the amplitude $\varphi$ a relative amplitude $\varphi-\varphi_{0}$ is the relovant quantity, we obtain the results shown in Fig. 12 where the upper curve is for a $12 \mathrm{sec}$ period and the lower curve is for a 4 sec period. The points correspond to the observed damping at an amplitude of $0.6 \mathrm{rad}$ for the $12 \mathrm{sec}$ period and 0.3 rad for the $4 \mathrm{sec}$ period. The curves were obtained by matching the data at $2.1^{\circ} \mathrm{K}$, which required $\epsilon=0.042$ for both periods. The result however is still in disagreement with observation, since the experimental points show that the excess damping is approximately independent of temperature and the theory predicts a strong temperature dependence. In summary, we find that the mutual friction force would give an excess decrement which is orders of magnitude larger than observed, and that even when the theory is adjusted to $\mathrm{fit}$ the data at $2 . \mathrm{l}^{\circ} \mathrm{K}$, the temperature dependence is incorrect. 
TABLE II

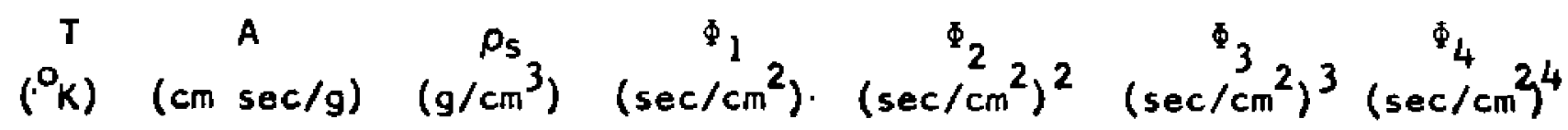

$\begin{array}{lllllll}2.1 & 190 & 0.03617 & 2.062 & 175.4 & 239.4 & 11,340\end{array}$

2.0

130

$0.0632 \quad 2.465$

44.22

$66.39 \quad 561.8$

$1.9 \quad 105$

0.0838

2.640

14.72

$22.24 \quad 49.25$

1.8

88

0.0999

2.637

6.340

7.477

5.697

1.6

60

0.1218

2.192

2.372

1.359

0.4973

1.4

38

0.1344

1.532

1.229

0.535

0.1113

1.3

28

0.1383

1.162

0.746

0.264

0.0439 


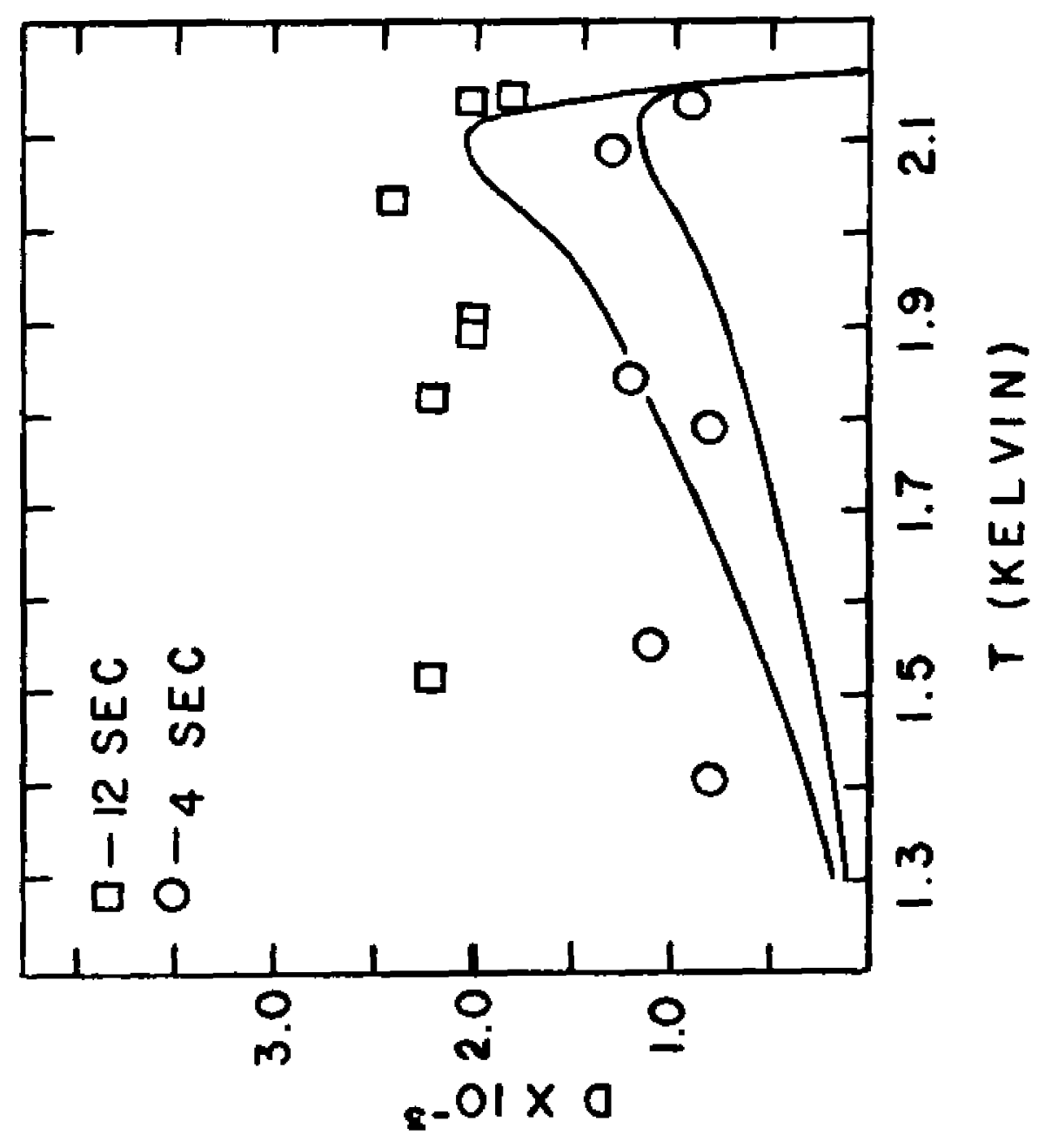

Fig. 12 
(2) Period Measurements

The relative change in added moment of inertia $I_{a}$ shown in Figs. 9 and 10 gives a measure of the amount of fluld being dragged by the disk. If the superfluid is "entrained" at high amplitudes, as suggested by Donnelly and Hollis-Hallett, ${ }^{6}$ then we would expect

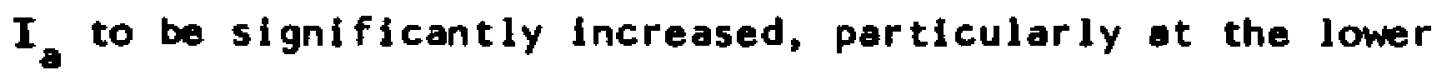
temperatures. Such behavior is indeed observed for the steep curves. If the effective viscosity is assumed to be approximately $\eta_{n}$, then the fraction of superfluid entrained can be calculated: the result for the data of Figs. 9 and 10 is $30 \%$ for the $3.9 \mathrm{sec}$ period at $1.52^{\circ} \mathrm{K}$ but only $5 \%$ for the $11.54 \mathrm{sec}$ perlod at $1.36^{\circ} \mathrm{K}$. The flat curves, on the other hand, show no evidence of entrainment and are In qualitative agreement with the results for classical 1iquids. At temperatures close to $T_{\lambda}$ the result for both steep and flat curves are consistent with the changes observed in ordinary liquids (dashed lines). Due to the fiber correction and the difficulty of determining $I_{a 0}$ precisely, the results shown in Figs. 9 and 10 are subject to large error, so only qualitative conclusions can be drawn. However, the minimum in $\Delta I_{a} / I_{\text {oo }}$ seen at the lower temperatures was observed repeatedly, so it is belleved to be a real effect, although its orlgin is obscure.

\section{v. SUMMARY AND CONCLUSIONS}

Our major conclusions ore: (1) that the damping curves and critical velocities observed in liquid hellum II with on oscillating disk are strongly dependent on the history of the hellum bath, and 
(2) that when the bath is not disturbed for about one hour after reaching $T_{\lambda}$, the resulting critical velocitles are independent of temperature and vary with period as $\tau^{-\frac{1}{2}}$.

We have conjectured that when the disk is set in motion shortly efter reaching $T \lambda$, the initial random motion of the superfiuid is organized into a long-lived state of motton. This long-lived state has the following features: (1) it does not interact with the low amplitude motion of the disk, (2) it causes the critical velocities to depend upon temperature and disk roughness, (3) it causes the superfluid to be entrained at high amplitudes, and (4) it persists as long as the temperature remains below $T_{\lambda}$. These results suggest that the long-lived state consists of a stable array of vortex lines and that the resulting critical velocities are of the non-ideal type.

When the disk is not deflected for one hour of ter reaching $T_{\lambda}$, the initial unorganized motion of the superfluld dies out and consequent measurements reveal the following features: (1) the critical velocities are higher than previously observed, are independent of temperoture, and (possibly) are Independent of disk roughness, and (2) the superfluld is not entrained at high amplitudes. Since the suberitical flow should be free of vorticity in this cese. the critfcal velocities ere presumed to be of the ideal type. From these critical velocities we obtain a temperature-independent kinematic viscosity, $\nu=1.2 \times 10^{-4} \mathrm{~cm}^{2} / \mathrm{sec}$, wich is then used to calculate criticel Reynolds number, Re - 72. This value is in good egreement with the value calculated from the Reynolds numbar 
proposed by Van Alphen et a1. 33 However, we reject the 1 dea that these critical velocities are due to turbulence in the normal fluld because (1) the excess demping is much larger than observed in ordinary liquids (see Fig. 3) and (2) no such critical value of the Reynolds number has been observed with oscillating disks In ordinary liquids.

The excess decrement at an amplitude slightly above critical was found to be temperature independent for the flat curves. A calculation of the excess decrement expected from mutual friction (based on a theory of Zwanikken) gave values which changed strongly with temperature and were much larger than observed.

The value of the low amplitude demping for both steep and flat curves was observed to increase when the bath haight fell to within about 100 penetration depths of the disk, suggesting a long range connection between energy dissipated in the vicinity of the disk and the loss of energy at the free surface of the hellum bath. This of fect does not occur In classicel liquids. 


\section{REFERENCES}

1. A. C. Hollis Hallett, Proc. Roy. Soc. A210, 404 (1952).

2. G. A. Gamtsemlidze, Zhur. Eksp. Teor. Fiz. S5R 34, 1434 (1958) [translation: Sov. Phys. JETP 37, 678 (1960)].

3. C. B. Benson and A. C. Hollis-Hallett, Can. J. Phys. 34. $668(1956)$

4. R. Dean Taylor and J. G. Dash, in Proceedings of the Fifth International Conference on Low Temperature Physics and Chemistry, edited by J.R. Dillinger (The University of Wisconsin Press, 1958) p. 164.

5. E. L. Andronikashvili, G. A. Gantsemlidze, and Sh. A. Dzhaparidze, J. Exp. Theo. Phys. (USSR) 50, 856 (1966) [translation: Sov. Phys. JETP, 23, 568 (1966)].

6. R. J. Donnelly and A. C. Hollis Hallett, Ann. Phys. 3 , $320(1958)$

7. H. E. Hall, Adv. Phys. (Phil. Mag. Suppl.) 2, 89 (1960).

8. E. J. Walker, Rev. Sci. Ins. 30, 834 (1959).

9. S. Rosenblat, J. FIuid Mech. 6, 206 (1959).

10. R. F. Folse, R. G. Hussey, and J.S. Bosnak, Phys. Fluids, $\underline{11}, 2508(1968)$.

11. A. D. B. Woods and A. C. Hollis Hallett, Can. J. Phys, 4l, $596(1963)$.

12. R. G. Hussey, B. J. Good and J. M. Reynolds, Phys. Fluids 10. $89(1967)$.

13. R. F. Folse and R. G. Hussey, Phys. Fluids 11, 2278 (1968). 
14. D. F. Brewer and D.0. Edwards, Proc. Roy. Soc. (London) A251. 247 (1959).

15. R. F. Folse, Unpublished.

16. J. G. Dash and R. D. Taylor, Phys. Rev. 105, 7 (1957).

17. W. F. Vinen, in Progress in Low Temperature Physics, edited by C. J. Gorter, vol. 2 (Amsterdam, 196!) p. 1.

18. L. Onsager, Suppl. Nuovo Cimento, 6,249 (1949).

19. R. P. Feynman, in Progress in Low Temperature Physics, edited by C. J. Gorter, vol. 1 (Amsterdam, 1955) p. 36.

20. D. F. Brewer, D. O. Edwards, and K. Mendelssohn, Phil. Mag. 1. $1130(1958)$.

21. D. F. Brewer and D. 0. Edwards, Phil. Mag. 6, 775 (1961).

22. K. Mendelssohn and W. A. Steelo, Proc. Phys. Soc. A73, 144 (1959) .

23. W. F. Vinen, Proc. Roy. Soc. A240, 128 (1957).

24. J. D. Reppy and C. T. Lane, Phys. Rev. 140, 106 (1965).

25. M. V. Ricci and M. Vicentinithissoni, Phys. Rev. 158, 153 (1967).

26. W. Vermeer, W. M. Van Alphen, J. F. Olijhoek, K. W. Taconis, and De Bruyn Ouboter, Phys. Letters 18, 265 (1965);

W. M. Van Alphen, J. F. Olijhoek, R. De Bruyn Ouboter, and K. W. Taconis, Physica 32, 1901 (1966).

27. W. E. Kelier and E. F. Hammel, Physics 2., 221 (1966).

28. K. Mendelssohn and G. K. White, Proc. Phys. Soc. A63, 1328 (1950). 
29. G. R. Hebert, K. L. Chopra, and J. B. Brown, Phys . Rev. 106, 391 (1957).

30. J. R. Clow and J. D. Reppy, Phys. Rev. Letters 19, 291 (1967).

31. J. S. Langer end M. E. Fisher, Phys. Rev. Letters 19, 560 $(1967)$.

32. W. F. Vinen, in Liguid Helium, edited by G. Careri (Academic Press, 1963) P. 336.

33. W. M. Van Alphen, G. J. Haasteren, R. De Bruyn Ouboter, and K. W. Taconis, Phys. Letters 20, 474 (1966).

34. G. C. J. Zwanikken, Physica 16, 805 (1950).

35. C. J. Gorter and J.H. Mellink, Physico 15, 285 (1949);

C. J. Gorter, P. W. Kasteleijn, and J. H. Mellink, Phys ica 16. $113(1950)$. 


\section{APPENDIX I}

\section{Calculation of Low Amplitude \\ Logari thmic Decrement}

The expression used to calculate the low amplitude logarithmic decrement is that $g i$ ven by Dash and Taylor: ${ }^{16}$

$$
\rho_{n}=\frac{R^{4} \pi\left[\pi \tau \eta_{n} \rho_{n}(1-D / 2 \pi)\right]^{\frac{1}{2}} k}{21\left(1+\tau^{2} / \tau_{v}{ }^{2}\right)}
$$

where $k=\left(1+2 d / R+3 \lambda d / R^{2}+8 \sigma \lambda / R\right)$ is a geometrical factor in which $\lambda$ is the normal fluid penetration depth, $R$ is the disk radius, $d$ is the disk thickness, and $\sigma$ is a corner parameter which was shown by Folse ${ }^{13}$ to have the value 0.30 . In making the actual calculations $0 / 2 \pi$ was neglected in comparison with unity and the ratio of the period to the vacuum period $\tau / \tau_{v}$ was assumed approximately equal to unity. The values of normal fluid viscosity $\eta_{n}$ and density $\rho_{n}$ were taken primarily from references 11 and 12 and are given in Table 111. Table IV contains (for selected temperatures and periods) the calculated value of the Low amplitude logarithmic decrement $D_{c a l c}=\left(D_{n}+D_{v}\right)$ as well as the observed value $D_{0}$, the height of the helium bath above the disk $h$, and the normal fluid penetration depth $\lambda$. 
TABLE II]

\begin{tabular}{|c|c|c|}
\hline $\begin{array}{l}T \\
\left({ }^{\circ} \mathrm{K}\right)\end{array}$ & $\begin{array}{c}\eta_{n} \\
(\mu P)\end{array}$ & $\underset{\left(g / \mathrm{cm}^{3}\right)}{p_{n}}$ \\
\hline 2.17 & 24.3 & 0.1427 \\
\hline 2.15 & 22.0 & 0.1296 \\
\hline 2.10 & 18.56 & 0.1097 \\
\hline 2.05 & 16.36 & 0.0951 \\
\hline 2.00 & 14.90 & 0.0825 \\
\hline 1.95 & 13.97 & 0.0713 \\
\hline 1.90 & 13.40 & 0.0617 \\
\hline 1.85 & 13.12 & 0.0532 \\
\hline 1.80 & 12.90 & 0.0455 \\
\hline 1.75 & 12.86 & 0.0389 \\
\hline 1.70 & 12.92 & 0.0333 \\
\hline 1.65 & 13.07 & 0.0281 \\
\hline 1.60 & 13.30 & 0.0235 \\
\hline 1.55 & 13.60 & 0.0194 \\
\hline 1.50 & 13.98 & 0.0160 \\
\hline 1.45 & 14.41 & 0.0133 \\
\hline 1.40 & 14.90 & 0.0108 \\
\hline 1.35 & 15.49 & 0.00883 \\
\hline 1.30 & 16.14 & 0.00690 \\
\hline 1.25 & 16.96 & 0.00545 \\
\hline 1.20 & 18.08 & 0.00419 \\
\hline 1.15 & 19.96 & 0.00308 \\
\hline 1.10 & 23.3 & 0.00220 \\
\hline
\end{tabular}


TABLE IV

$\begin{array}{lcccccc}\mathrm{T}^{\mathrm{N}} & \mathrm{T} & \mathrm{h} & \lambda & \mathrm{h} / \lambda & \mathrm{O}_{\mathrm{o}} & \mathrm{D}_{\mathrm{cal}} \mathrm{c} \\ \left({ }_{\mathrm{K}}\right) & (\mathrm{sec}) & (\mathrm{cm}) & (\mathrm{cm}) & & & \\ 2.091 & 3.9 & 13.02 & 0.0143 & 910.3 & 0.0125 & 0.0125 \\ 1.846 & 3.9 & 12.38 & 0.0174 & 711.6 & 0.0075 & 0.0075 \\ 2.037 & 11.54 & 17.15 & 0.0251 & 683.1 & 0.01890 & 0.01890 \\ 2.146 & 8.84 & 14.61 & 0.0214 & 682.5 & 0.00460 & 0.00460 \\ 2.045 & 19.5 & 16.83 & 0.0324 & 519.4 & 0.0258 & 0.0257 \\ 1.824 & 11.54 & 12.7 & 0.0309 & 411.0 & 0.01265 & 0.01260 \\ 1.556 & 3.9 & 9.53 & 0.0290 & 328.4 & 0.0049 & 0.0047 \\ 1.518 & 11.54 & 9.84 & 0.054 & 182.0 & 0.0084 & 0.0079 \\ 1.408 & 3.9 & 6.67 & 0.0405 & 164.6 & 0.0043 & 0.0041 \\ 1.433 & 19.6 & 11.75 & 0.0851 & 138.04 & 0.0100 & 0.00915 \\ 1.413 & 11.54 & 8.89 & 0.0679 & 130.9 & 0.0076 & 0.0067 \\ 1.75 & 45.0 & 8.92 & 0.0684 & 130.0 & 0.00915 & 0.00845 \\ 1.825 & 12.4 & 3.81 & 0.0317 & 120.0 & 0.0140 & 0.0133 \\ 1.475 & 8.84 & 5.72 & 0.0520 & 109.9 & 0.0022 & 0.00173 \\ 1.94 & 45.0 & 5.08 & 0.053 & 95.85 & 0.0132 & 0.01115 \\ 1.582 & 19.1 & 5.25 & 0.0603 & 86.9 & 0.0130 & 0.0114 \\ 1.33 & 3.9 & 3.81 & 0.049 & 77.7 & 0.0019 & 0.0014 \\ 1.429 & 19.6 & 5.87 & 0.0860 & 68.3 & 0.0120 & 0.0091 \\ 1.346 & 9.33 & 3.81 & 0.0727 & 52.4 & 0.0345 & 0.00230 \\ 1.25 & 19.1 & 4.13 & 0.1409 & 29.3 & 0.0120 & 0.0072 \\ 1.415 & 19.6 & 2.22 & 0.0886 & 25.1 & 0.0130 & 0.0089 \\ 1.488 & 12.1 & 1.27 & 0.0591 & 21.5 & 0.00325 & 0.00183\end{array}$




\begin{abstract}
APPENDIX II
Computer Program Used to Calculate

and Plot Logarithmic Decrement Curves
\end{abstract}

An IBM 360 Mod 65 computer was used to compute the logarithmic decrements from the data taken in the lab, and in conjunction with the computer a Calcomp 563 incremental plotter was used to plot the curves of logarithmic decrement versus amplitude. The plotter is coupled to a Calcomp 570 magnetic tape unit, and information required to drive the plotter is recorded on a magnetic tape by means of fortran IV routines available on the 360 computer.

A typical program used in the calculation of $D$ for disk $C$ with a 13 second period is listed below:

C LOG DECREMENT PROGRAM FOR 12.87 SEC PERIOD 7/1/69

DIMENSION DATA (2000)

CALLPLOTS(DATA $(1), 2000)$

DIMENSION A(900),W(60), CRV $(60), D O(9), A O(9)$

$N T=1$

$\operatorname{READ}(5,100) \mathrm{NB}$

100 FORMAT (13)

$\operatorname{READ}(5,300)(\operatorname{CRV}(1), 1=1, N B)$

300 FORMAT (18F4.1)

$\operatorname{READ}(5,200)(W(N), N=1, N B$

$\operatorname{READ}(5,200)(D O(N), N=1, N B)$

$\operatorname{READ}(5,200)(A O(N), N=1, N B)$

200 FORMAT (12F6.3)

$N=0$ 
$5 \operatorname{CALLPLOT}(0,0,0.0,+3)$

CALLPLOT $(0.0,16.0,2)$

CALLPLOT $(17.0,16.0,2)$

CALLPLOT $(17.0,0.0,2)$

$\operatorname{CALLPLOT}(0,0,0.0,2)$

CALLPLOT $(0.5,0.5,-3)$

CALLAXIS $(0.0,0.0$, 9HDECREMENT, 9, 15.0,90.0,D0(NT), 0.004,5.0)

4 CALLAXIS $(0.0,0.0,9$ HAMPLITUDE, $-9,16.0,0.0, A O(N T), 0.15,5.0)$ GO TO 8

$6 N T=N T+1$

IF(N.EQ.NB)GO TO 25

3 IF (CRV (NT) .EQ.NT) GO TO 7

GO TO 8

7 CALLPLOT $(-0.5,-0.5,-3)$

CALLPLOT $(18.0,0.0,-3)$

GO TO 5

$8 \mathrm{~N}=\mathrm{N}+1$

$k=-11$

$L=0$

$15 \quad K-K+12$

$L=L+12$

$\operatorname{READ}(5,10)(A(1), 1=K, L)$

IF(A(L) -0.0$) 16,16,15$

10 FORMAT (12F6.3)

$16 \mathrm{~J}=3$

$1=0$ 


$$
\begin{aligned}
& 17 Y=7.00 \\
& 51 \quad Y=Y-0.50 \\
& J=J+1 \\
& 40 \quad 1=1+1 \\
& \mathrm{X}=\mathrm{J} \\
& M=I+J \\
& \text { IF (A(M).EQ.0.0)GO TO } 6 \\
& Q=A(1)-A(M) \\
& Z=W(N) \\
& A l=(A(1)-Z) * 0.015 \\
& A 2=(A(M)-Z) \div 0.015 \\
& \text { I } F(Q-Y) 50,60,60 \\
& \text { so } 1=1-1 \\
& \text { GO TO } 51 \\
& 60 B=(A 1+A 2) / 2,0 \\
& D=A \operatorname{LOG}(A 1 / A 2) / X \\
& 20 \text { WRITE }(6,23) \mathrm{J}, Y, B, D \\
& 23 \text { FORMAT }(1 X, 16,2 F 9.3, E 16.8) \\
& \operatorname{IF}(D-D O(N T)) 40,40,42 \\
& 42 B=(B-A O(N T)) * 6.666666 \\
& D=(D-D O(N T)) * 250.0 \\
& \text { IF (N.EQ.1) } N J=0 \\
& \text { IF (N.EQ.2) } N J=1 \\
& \text { IF(N.EQ.3) } \mathrm{NJ}=2 \\
& \text { IF(N.EQ.4) } \mathrm{NJ}=5 \\
& \text { IF (N.EQ.5) NJ=14 }
\end{aligned}
$$




$$
\begin{aligned}
& \text { IF(N.EQ.6) NJ=92 } \\
& \text { IF(N.EQ.7) NJ=3 } \\
& \text { IF }(N . E Q .8) \quad N J=0 \\
& 43 \text { CALLSYMBOL }(B, 0,0.10, N J, 0.0,-1) \\
& \text { GO TO } 40 \\
& 25 \text { CALLPLOT }(0.0,0.0,-3) \\
& \text { CALLPLOT }(0.0,0.0,999) \\
& \text { STOP } \\
& \text { END }
\end{aligned}
$$

The number NB is the total number of curves to be analyzed. The subscripted variable $C R V(1)$ is employed in statement 3 to determine how many curves will be plotted on a given set of axes, i.e. if after plotting 2 curves on the first set of axes 1 want to draw another set before plotting the third curve then CRV (3) $=003.0$. The zero correction for each run is read in as $W(N)$ and both $D O(N)$ and $A O(N)$ are the values of the logarithmic decrement and amplitude respectively at the origin of the axes for a given curve N. After drawing the axes (statements 5-4) the data is read in (statements $8-10$ ). The number 00.000 is placed in the last position of the last card for each run to ensure that only one run is read in at a time. Statements 16, 17, and 51 regulate the initial choice of the number of amplitudes between those being used to calculate $D$ and the final number to be used, which should be predetermined by the size of the damping. Each $\log$ decrement $D$ and corresponding amplitude $B$ is then computed, printed out, and plotted up one at a time. In plotting each point up (statement 43) NJ 
refers to a code number which corresponds to the particular symbol to be used, and both $B$ and $D$ are converted to inches (statement 42 ). 
VITA

Robert Patrick Roger was born in New Orleans, Louisiana on May 21, 1942. He attended elementary and high schools in New Orleans and was graduated from Jesuït High School in 1960 . He entered Loyola University of the South in New Orleans, in September, 1960 and received a Bachelor of Science degree in physics in 1964. He entered the Graduate School of Louisiana State University in September, 1964, held an N.D.E.A. fellowship in the Department of Physics and Astronomy during his first three years of graduate study, and served as a graduate research assistant in the same department from September 1968 to the present. He is presently a candidate for the degree of Doctor of Philosophy in the Department of Physics and Astronomy. 
EXAMINATION AND THESIS REPORT

Candidate: Robert Patrick Roger

Major Field: Physics

Title of Thesis: Amplitude Effects for the Oscillating Disk in Liquid Helium II

Approved:

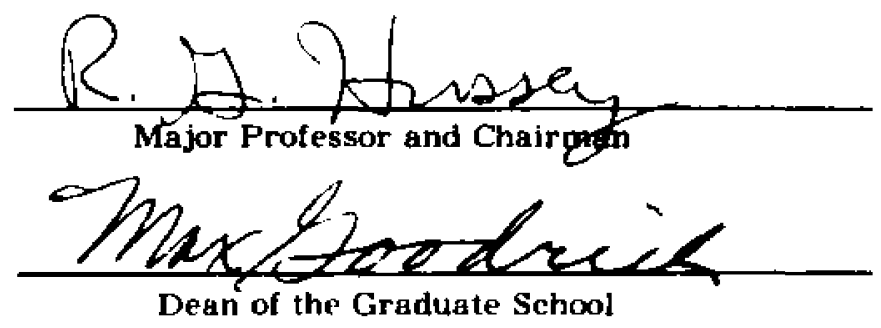

EXAMINING COMMITTEE:
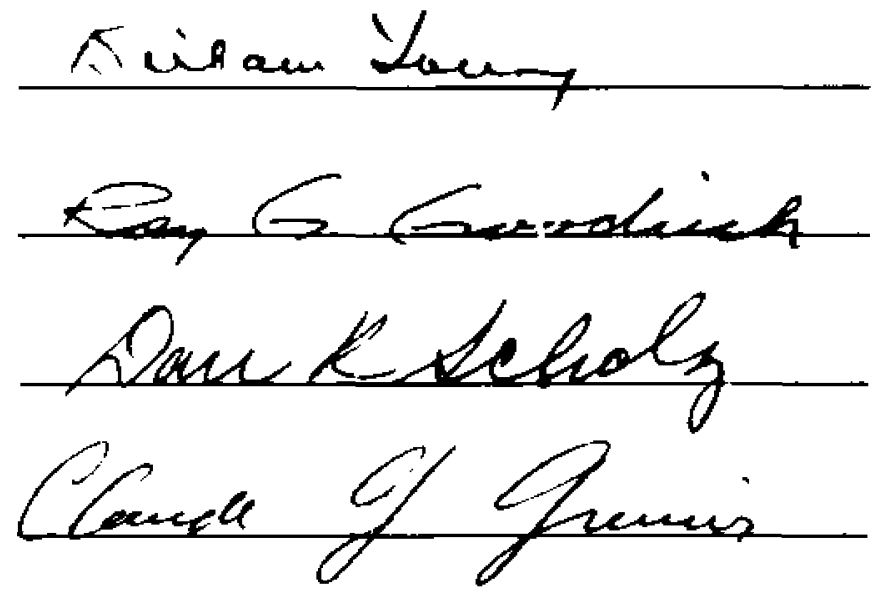

Date of Examination:

July 25, 1969 